\title{
Complexities of Biosimilar Product
}

\section{Alok Bandyopadhyay*}

Consultant Design Space Inpharmatics, USA

\begin{abstract}
Biosimilar Act, passed in 2007 under 351(k), states that a biosimilar product should be "highly similar" to prior approved reference product (RLD) and will have "no clinically meaningful differences" in their safety or efficacy. FDA also published three guidances to support this biosimilar project. However, the biosimilar processes are not so smooth. The biological molecules, manufacturing processes, and impurities profiles are complex; leaving various issues in deriving different sections of CMC work. Due to relative complexities in producing biosimilar product small differences in the design and execution of manufacturing process can have a large influence of product related-, process related-,or host related impurities protein profile of a finished product, which may trigger immunogenicity and changes the clinical profile requiring elaborate animal studies and human clinical studies. FDA's guidance is not very clear to drive the product into regulatory pathway for approval. Complexities still exist in clinical studies as clinicians like to review the details of data from three phase 3 trials and payors wish to see more stringent data regarding safety and efficacy. However, EMA developed a centralized path for the approval of Biosimilar product and so far EMA approved 16 products.

One of the remedies is that if the biosimilar product is purified to homogeneity or near homogeneity, and if it is stabilized and restores the functional activities, the impurities protein content will be negligible or minimum, which may not trigger the immunogenicityor other clinical issues. With more advancement of science, research and development may solve these issues and open the easier regulatory pathway for biosimilar approval. Interchangeability and price reduction issues may be solved at that time.
\end{abstract}

\section{Keywords: EMA; Biosimilar; Glycosylation}

\section{Introduction}

The Biosimilars Act was passed as part of the recent Patient Protection and Affordable Care Act.66. Under $₫ 351(\mathrm{k})$ of the Biosimilars Act (“351(k) application"), the law states that a biosimilar manufacturer must demonstrate that its product is similar to a prior approved reference product [1]. In order to meet this standard, the statute requires that an applicant must show that the two products are "highly similar" and have "no clinically meaningful differences" in their safety or efficacy. Neither "highly similar" nor "no clinically meaningful differences" are well defined in statute. In the meantime FDA developed the guidance in order to drive the product into development processes for regulatory pathway to approval [2,3]. Similar guidance was published by EU and they approved a few drugs on the basis of their guidance [4]. The purpose of this article is to discuss the complexities of biological molecules and also the complexities of its drug development procedures along with FDA guidance.

\section{Complexities of the Biological Molecules}

Most small molecule drugs with molecular weight ranging from one hundred to several hundred times the weight of hydrogen atom are produced through some well-established chemical synthetic steps and purified through a rigorous process. This enables the production of substantially pure form of drug substances or Active Pharmaceutical Ingredient (API). This API was characterized further by various physical and chemical methods [5].

In contrast, biological molecules are proteins or peptides composed of several amino acids. The molecular weight of these biologics varies from several hundred to several thousand times the weight of hydrogen atom. The structure and conformations of these molecules are also complex as proteins exist in three types of structure: primary, secondary and tertiary.

Protein folding is a very important step to convert primary structure to secondary structure. Further it interacts with different type of structures (e.g. tertiary, quaternary) to develop its stability and therapeutic values. Several factors are involved in protein folding including enzymatic interactions and the chemistry present in hosts. The subsequent purification, processing, and storage of such a protein may affect its structure or alter the function of a protein which may be related to the safety and efficacy of a drug [6,7].

In addition post-translational modification occurs when enzymes present in a cell add various other molecules to the protein structure of the drug. The addition of these other molecules includes, for example, sugars (glycosylation) and phosphate groups (phosphorylation) [8]. Like structural changes, post-translational modification can affect the function, stability, bioavailability, and immunogenicity of a protein. Improper glycosylation of human erythropoietin or missing one or more glycosylation site may reduce the drug's intended effect [9-13].

\section{Complexities in Manufacturing}

Small molecule pharmaceutical drugs, produced by chemical synthesis, have uniform predictable structure and are generally stable. In contrast, biological drugs are protein based and large in molecular size. They are produced through living organism. As described earlier their structures are complex and require specific conditions for stability.

Biological drug is manufactured through recombinant DNA

*Corresponding author: Alok Bandyopadhyay, Consultant Design Space Inpharmatics, USA, E-mail: akbandy@gmail.com

Received September 21, 2013; Accepted October 29, 2013; Published October 31, 2013

Citation: Bandyopadhyay A (2013) Complexities of Biosimilar Product. J Bioanal Biomed 5: 118-121. doi:10.4172/1948-593X.1000091

Copyright: (c) 2013 Bandyopadhyay A. This is an open-access article distributed under the terms of the Creative Commons Attribution License, which permits unrestricted use, distribution, and reproduction in any medium, provided the original author and source are credited. 


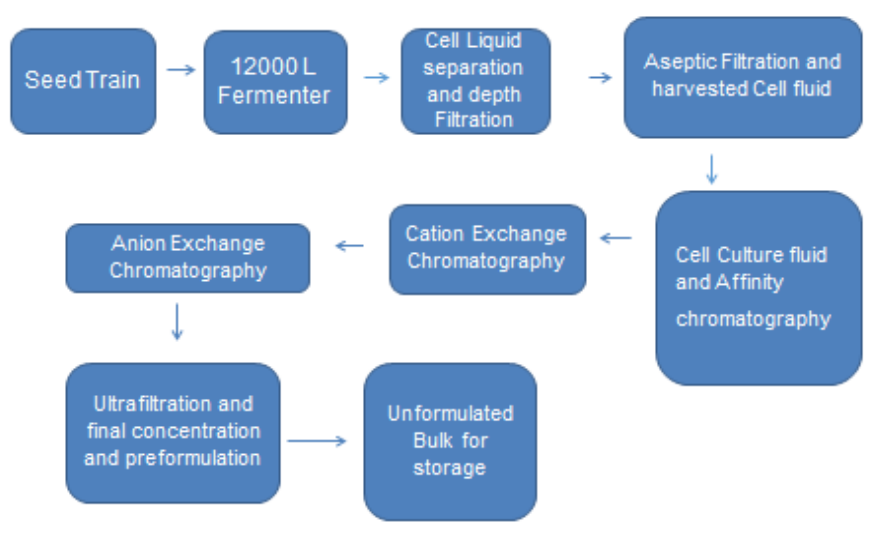

Figure 1: Manufacturing of biological products.

technology and is a highly complex procedure [14]. It starts with the modification of a cell or microorganism, considered as host to introduce a genetic sequence coding for the desired protein. Once the right sequence for the protein is introduced, the host is conserved and produced master cell bank (MLB), from which a seed lot is picked up, cultured and grown in a bioreactor or fermenter.Then it is subjected to multistep of harvesting. Even at this point the desired protein is contaminated with several other impurities. So it is required to purify the protein in a fairly pure state. Once the purified protein is obtained, it is stabilized and then formulated for use as a therapeutic drug. A typical biological manufacturing process was shown in Figure 1.

During the various steps of manufacturing e.g. cells grown, harvest and the multistep purification, one has to examine that the protein moiety preserves the biological activity for use of a particular therapeutic indication. So the manufacturing processes and CMC (chemistry manufacturing controls) information are keys to the biological drug development. Such information includes source material, individual process steps in growing cells, harvest, protein purifications, sterile filling and drug product formulation. Any change in the processes e.g. excursion of temperature, $\mathrm{pH}$ of media, cell culture condition, or even storage and transport may cause partial or full loss of biological activity or enhanced biological activity which will result automatically in significant difference in the outcome of clinical testing e.g. safety and efficacy of the biological drug. Both neutralized biological activity as well as enhanced potency can bring serious safety issues [14-16]. Replacing stabilizer serum albumin from Eprex (epoetin product) with polysorbate 80 to become compliant with EU regulation caused severe immunogenicity response. After thorough investigation it was found the interaction between polysorbate 80 with rubber stopper caused pure red cell aplasia. Ultimately manufacturer rectified this issue [16].

\section{Complexities in Impurity Profile}

Unlike small molecule pharmaceuticals, it is difficult to purify biological products to homogeneity.Due to the larger size and complexity of protein based drug, it is difficult to synthesize chemically. As mentioned earlier the folding and structural conformations play a big role in demonstrating drug activity. In order to restore drug activity in the biological product, the biological molecule is purified through several steps and exposed to several biomolecules which may contaminate biological product with other materials. During the purification or preservation processes, the protein molecule may be oxidized or deaminated or deamidated. If the product is manufactured in different host; the product may be contaminated with host related impurities. So the contamination may be due to:

- $\quad$ product related impurities,

- process related impurities,

- host related or other contaminates.

Again the testing methods are product specific and proprietary, so each manufacturer has to develop product related methods, which may be different from the branded product. Similarly, they are required to prepare their own standards. During purification, the product may remain as monomer, dimer or other conformations. The effectiveness of the different conformation of the molecule is required to determine with respect to safety. In addition, host related or cell substrate derived impurities are required to develop the testing methods and purified the product to a minimum content of host derived proteins.

The method of purification of biological drug consists of several steps, so the biological products may be contaminated with process related materials either from upstream processes or downstream processes. Again, the content of these materials has to be reduced to minimum.

Other impurities e.g. endotoxin, bioburden, heavy metals, Arsenic are required to be tested and are required to reduce to its minimum. In addition, the absence of any adventitious agents in unprocessed Bulk has to be demonstrated.

Changes in a protocol at any of the manufacturing steps can have important consequences for final product. For example, depending on the conditions in which a product is expressed, it may contain molecular artifacts, proteins, or other impurities from the host cell and may be differentially oxidized or glycosylated. These differences may increase the product's immunogenicity, and can produce hypersensitivity reactions. This may alter the products pharmacokinetic and pharmacodynamics (PK/PD) profile.

\section{Complexities in Deriving CMC Work}

The CMC information for each biological drug is so specific; manufacturers often develop product specific proprietary standards, test and various other assays during the development. They maintained these procedures throughout the life-cycle to ensure the standard of the biological product which are used in biological drug. Since the CMC information is proprietary, it is difficult for other manufacturers to produce the product in the same standard as originator. So the knowledge and experience with these proprietary standards, tests and assays are essential to maintain the same standard of the products as originator.

Biosimilar manufacturer produces the drug product in a different protocol from the innovator's drug manufacturing protocol. Such protocol variations may have a significant impact on the production of drug products. So the regulatory agencies have established a number of similarity metrices in order to control these variations.. Moreover, the high degree of similarity between the innovator's drug and biosimilar products may probably be achieved, but small differences in the design and execution of a manufacturing process can have a large influence on the clinical profile of a final finished product, raising the need for careful design of manufacturing quality-control processes. If sponsor produces exact copy of the innovator's protein sequence (primary structure) in the drug product, but it may still be lacking proper conformations (e.g. secondary, tertiary or quaternary structure). Both 
the molecular size and the structural variations resulting from protein folding and post-translational modifications also contribute towards complexities.

Due to the greater size and complexity of protein-based drugs, they often cannot be synthesized by chemical synthetic steps. Instead they are produced in various living organisms e.g. bacteria, yeast, plant or mammalian cell cultures. So it is difficult to maintain consistency of production processes. Sometimes natural variations and changes in host living organisms may cause some discrepancies between batches to batches including mutations in the DNA of the host, which may change the protein sequences of the drug.

Alteration of protein structure and introduction of impurities can readily occur during formulation, storage, and delivery, with potentially serious effects on potency and safety. This raises the importance of careful review of a biosimilar product's safety, efficacy, and manufacturing during the approval process, as well as of continued assessment of its production protocols in post-approval. Furthermore, even if biological manufacturing process has been perfected in a small scale, but scaling may provide several challenges.

\section{Complexities in Performing Clinical Work}

Complexities exist in clinical work as Clinicians like to review biosimilar's action profile, storage, stability, expiration once in use, predictability of absorption from injection site, and bioavailability to build up their confidence limit for prescription to patients $[17,18]$.

Meanwhile, a Bio Trends Research Group survey reported that a group of doctors prefer to review the data from 3 phase III clinical studies, immunogenicity study, preclinical comparability assessment and approved manufacturing methods. Moreover, payor like to review the clinical study data of patients treated with biosimilar products.

\section{Complexities in Immunogenicity}

As mentioned earlier in impurity profile that protein impurities generated from product, process or host related, can increase immunogenicity making biological drugs especially vulnerable to immunogenicity issues $[11,17]$. Moreover, the patients develop various antibodies following the treatment with biological drugs and it may sometimes produce system translational modification of proteins $[12,18]$.

\section{Complexities in FDA Guidance}

Although the Biosimilars Act, passed by congress as a part of the recent Patient Protection and Affordable Care Act.66, creates an abbreviated approval pathway for biological products or biological licensing applications (ABLA)(1). Like small molecule pharmaceuticals, biological product can be shown to be sufficiently similar to a previously approved biological drug. In order to clarify and expedite the process, FDA recently published guidance (2-4). These guidances state the following:

1. A comprehensive structural analyses of the protein based drug along with the development of a functional assay for the measurement of potency

2. In addition, sponsor should perform animal studies to test the toxicity, pharmacokinetics, pharmacodynamics, and immunogenicity of the sponsored drug

3. In order to demonstrate that "there are no clinically meaningful differences between the biological product and the reference product in terms of the safety, purity, and potency of the product", there is a need for human clinical trial.

In case if there are "residual uncertainties" about the biosimilarity of a drug, more clinical studies are required for establishing safety and efficacy, of that particular drug.

\section{Biosimilar Products Approval in EU}

The first guidance was issued by European Medicines Agency (EMA) sometimes in 2004 and the first product, Omnitrope (somatropin, was approved in 2006. Due to complexities of biological drugs, many of the concepts discussed in the guidance might have to be adopted on a case by case basis. In addition to general guidances, four product class-specific guidelines were issued by EMA for the development of Biosimilars containing epoetin, somatotropin, human insulin, and human granulocyte colony stimulating factors. These guidelines also discussed the preclinical and clinical requirements for marketing approval of Biosimilars in EU. At present EMA developed a path for approval of Biosimilars, which is a centralized procedure. Subsequently EMA published several general and specific guidelines for Biosimilars. So far they (EMA) approved 16 Biosimilar products [19]. All of these products are not available in the market and the safety of these products was not established through pharmacovigilance studies.

\section{Proposal for Remedies}

As described earlier, the drug development for biosimilar product is complex.Starting from CMC of the biological molecule to its clinical development. Moreover, the guidance is not clear in the development of regulatory pathways. Several remedies may be available in future. But at the present time two remedies can be thought of as described in the following:

First is the development of purification procedure of the biosimilar product to homogeneity or near homogeneity, and if it is stabilized with the preservation of the functional activities and no toxicological issues, the impurities protein content will be negligible or minimum. Such a low content of protein impurity may not trigger the immunogenicity or other clinical issues. With more advancement of science, research and development may solve these issues in the near future. This may open the easier regulatory pathway for biosimilar approval. Interchangeability and price reduction issues may be solved at that time.

Other remedy is the regulatory path of faster approval of biosimilar products. Priority review program of FDA, established in 1992, reduced the review time from 10 months to 6 months. Other paths e.g. fast track and accelerated approval still exist. Recently FDA adopted a new path of faster approval for innovative drugs designed as Breakthrough therapy through collaboration between FDA and manufacturer, which is applicable for (a) Life threatening or serious illness (b) Early clinical trial shows better efficacy over existing therapies.Biosimilar also requires a similar path for faster approval.

\section{Conclusion}

Biosimilar Act, passed in 2007 under 351(k), states that a biosimilar product should be "highly similar" to prior approved reference product and will have "no clinically meaningful differences" in their safety or efficacy. It was thought at that time that the biosimilar product would be manufactured from an optimum stable highly productive cell-line and 
characterized by testing with developed analytical methods, enhancing the production of a small scale GMP lot for the animal studies (PK, $\mathrm{PD})$, toxicology and more comprehensive comparability studies with reference listed drug (RLD). This will finalize the release testing, stability indicating methods, analytical validations, manufacturing including in process, and scale up manufacturing promoting the performance of PK/PD study, clinical immunogenicity studies, clinical trial studies, and continued biochemical/biological comparability studies from the registration batches. But the biosimilar processes are not so smooth. The biological molecule, manufacturing processes, and impurities profile are complex.The protein impurities from product related, process related or host related may trigger immunogenicity requiring elaborate animal studies and human clinical studies. FDA's guidance is not very clear to drive the product into regulatory pathway for approval, whereas EMA developed a centralized path for the approval of Biosimilar product and so far EMA approved 16 products.

One of the remedies is that if the biosimilar product is purified to homogeneity or near homogeneity, and if it is stabilized with preservation of the functional activities, the impurities protein content will be negligible or minimum, which may not trigger the immunogenicity or other clinical issues. With more advancement of science, research and development may solve these issues and open the easier regulatory pathway for biosimilar approval. Interchangeability and price reduction issues may be solved at that time. On the other hand, biosimilar products require faster approval. Probably the Agency will develop the quicker approval process in the near future.

\section{References}

1. http://www.gpo.gov/fdsys/pkg/PLAW-111publ148/content-detail.html

2. http://www.fda.gov/downloads/Drugs/GuidanceComplianceRegulatoryInformation/Guidances/UCM291128.pdf

3. U.S. Food and Drug Administration (2012) Guidance for Industry on Biosimilars: $\mathrm{Q} \&$ As Regarding Implementation of the BPCI Act of 2009.
4. http://www.ema.europa.eu/docs/en_GB/document_library/Scientific guideline/2012/05/WC500127960.pdf

5. Rick NG (2009) Manufacture of Large Molecule APIs (Recombinant DNA Methods). ( $2^{\text {nd }}$ Edn), Wiley-Blackwel, Hoboken, NJ, USA 340-348.

6. Dimasi JA (2001) New Drug Development in the United States from 1963 to 1999. Clin Pharmacol Ther 69: 286-296.

7. Khoury GA, Baliban RC, Floudas CA (2011) Proteome-wide post-translational modification statistics: frequency analysis and curation of the swiss-prot database. Scientific Reports.

8. www.piercenet.com/browse.cfm?fldID=7CE3FCF5-0DA0-4378-A513

9. Larsen MR, Trelle MB, Thingholm TE, Jensen ON (2006) Analysis of posttranslational modifications of proteins by tandem mass spectrometry. Bio Techniques 40: 790-798.

10. Jenkins N, Murphy L, Tyther R (2008) Post-translational modifications of recombinant proteins: significance for biopharmaceuticals. Mol Biotechnol 39: 113-118.

11. Delorme E, Lorenzini T, Giffin J, Martin F, Jacobsen F, et al. (1992) Role of glycosylation on the secretion and biological activity of erythropoietin. Biochemistry 3: 9871-9876.

12. (2011) Shaping the biosimilars opportunity: A global perspective on the evolving biosimilars landscape. IMS Health.

13. Manufacture of Biological Medicinal Products for Human Use.

14. Spiker B (2009) Guide to Drug Development: A Comprehensive Review and Assessment. Wolters Kluwer Health \&Lippincott Williams \& Wilkins, 119-131.

15. Rick NG (2009) Drugs: From Discovery to Approval.John Wiley \& Sons, Canada 93-135.

16. Michael Hay, Jesse Rosenthal, David Thomas, John Craig (2011) Bio/Biomed tracker clinical trial success rates study, BIO CEO \& Investor Conference,BIO.

17. Class JN, Langis L (2012) A patient-centered paradigm for the biosimilars market. Communications 1: 17-21.

18. Keeping K (2012) Biosimilar opportunity within the insulin market. Presentation at GTC Bio 2nd Annual Diabetes Summit,Boston, USA, 19-20.

19. Biosimilars approved in Europe, Generics and Biosimilars Initiatives. 